\title{
Is There a Sex Difference in Japanese Patients With Heart Failure?
}

\author{
Shinichiro Suna, MD, PhD; Keiko Yamauchi-Takihara, MD, PhD
}

$\mathbf{H}$ eart failure (HF) is a leading cause of cardiovascular mortality and morbidity both in Japan and the Western world. ${ }^{1}$ In the United States, HF incidence has largely remained stable over the past several decades, with $>650,000$ new HF cases diagnosed annually, approximately 5.1 million persons with clinically manifest $\mathrm{HF}$, and the prevalence of HF continuing to rise. ${ }^{2}$ In 2008, the Ministry of Health, Labor and Welfare, Japan, estimated that there were 47,500 patients with $\mathrm{HF}$ and 27,900 patients hospitalized for this disease per day in this country. ${ }^{3}$ The incidence of HF increases with age, rising from approximately 20 per 1000 individuals aged 65-69 years to $>80$ per 1000 individuals among those $\geq 85$ years old. In 2011, the number of deaths from HF was 69,368 (26,011 males, 43,357 females), and death rate (per 100,000 population) was 55.0 (42.3 in males, 67.0 in females).$^{4}$

\begin{tabular}{l} 
Article p 428 \\
\hline HF affects men at a younger age than women. Women more \\
frequently have diastolic HF, associated with the major risk \\
factors of diabetes and hypertension, and men more frequent- \\
ly have systolic HF because of ischemic heart disease. ${ }^{1}$ Meta- \\
analyses from large multicenter trials confirm that women are \\
less likely to receive guideline-related HF treatment, but nev- \\
ertheless do better than their male counterparts. ${ }^{5}$ Martínez- \\
Sellés et al performed the meta-analysis of sex differences in \\
HF using the Meta-Analysis Global Group In Chronic Heart \\
Failure (MAGGIC), and revealed that survival is better for \\
women with HF compared with men, irrespective of ejection \\
fraction (EF). Furthermore, this survival benefit is more marked
\end{tabular}

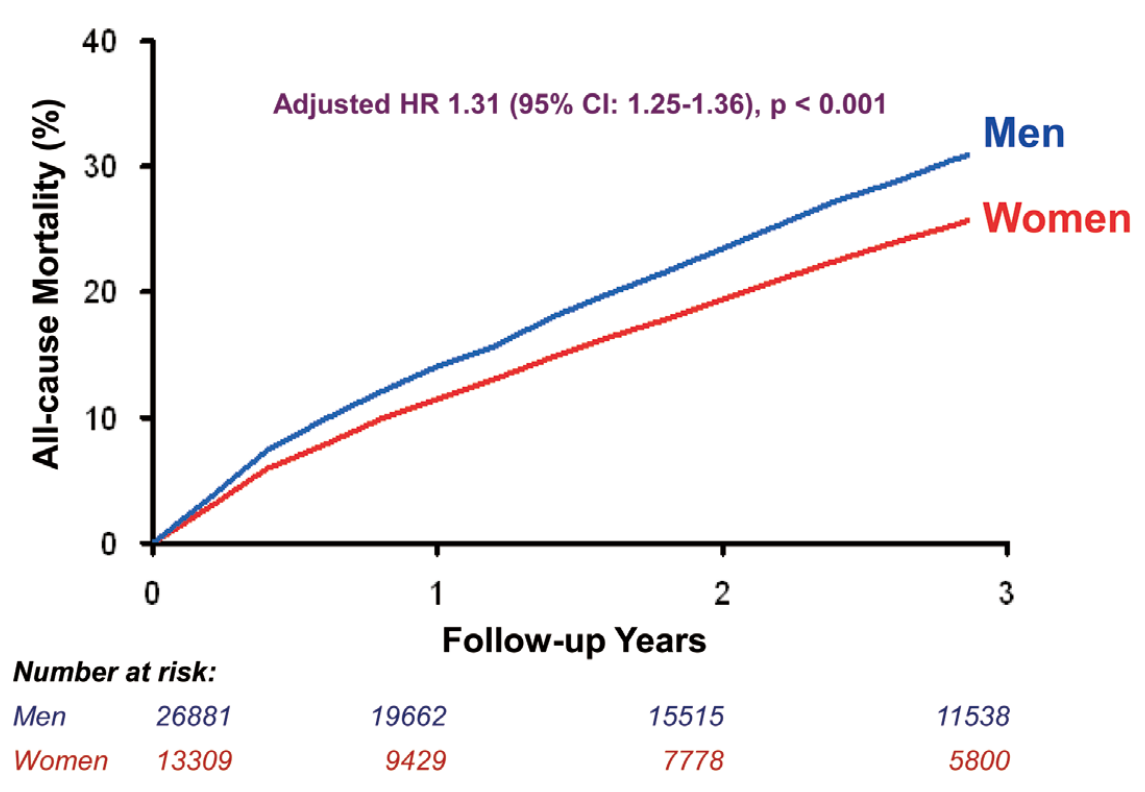

Figure. All-cause mortality for men and women adjusted for age. The risk of death is higher in men than in women with heart failure when adjusted for age (hazard ratio (HR) 1.31, 95\% confidence interval (Cl) 1.25-1.36, $\mathrm{P}<0.001$ ). (Adopted from MartínezSellés et al. ${ }^{6}$ )

The opinions expressed in this article are not necessarily those of the editors or of the Japanese Circulation Society.

Received December 12, 2013; accepted December 12, 2013; released online December 25, 2013

Department of Cardiovascular Medicine, Osaka University Graduate School of Medicine, Suita (S.S., K.Y-T.); Health Care Center, Osaka University, Toyonaka (K.Y-T.), Osaka, Japan

Mailing address: Keiko Yamauchi-Takihara, MD, PhD, Health Care Center, Osaka University, 1-17 Machikaneyama, Toyonaka, Osaka 560-0043, Japan. E-mail: takihara@wellness.hss.osaka-u.ac.jp

ISSN-1346-9843 doi:10.1253/circj.CJ-13-1507

All rights are reserved to the Japanese Circulation Society. For permissions, please e-mail: cj@j-circ.or.jp 
in non-ischemic HF. ${ }^{6}$ Data from 31 studies (41,949 patients; 28,052 men, 13,897 women) from the MAGGIC showed that women were older $(70.5 \pm 12.1$ vs. $65.6 \pm 11.6$ years $)$, more likely to have a history of hypertension $(49.9 \%$ vs. $40.0 \%)$, and less likely to have a history of ischemic heart disease $(46.3 \%$ vs. $58.7 \%)$ and reduced EF (62.6\% vs. $81.6 \%)$ compared with men. During a 3-year follow-up, 3,521 (25\%) women and $7,232(26 \%)$ men died, which was comparable. After the adjustment for age, male sex was an independent predictor of mortality (Figure), and the better prognosis associated with female sex was more marked in patients with HF of nonischemic etiology, compared with ischemic etiology. ${ }^{6}$

$\mathrm{HF}$ and preserved EF (HFpEF) constitutes $30-50 \%$ of $\mathrm{HF}$ patients, and affects women more often than men. Deswal et al evaluated the sex difference in mortality and morbidity for HFpEF (EF $>50 \%$ ) using the ancillary arm of the Digitalis Investigation Group (DIG) trial. They concluded that, although the clinical manifestations of HF appear to be more severe in women with HFpEF, after adjustment for baseline clinical differences, HF hospitalizations are not increased and survival expectancy is better for women than men. ${ }^{7}$

In this issue of the Journal, Sakata et $\mathrm{al}^{8}$ used the CHF Registry in Tohoku area of Japan, named CHART-2 Study and show that female chronic HF (CHF) patients had better survival than males after adjustment for baseline differences, although the crude mortality rate was comparable between the sexes, possibly reflecting relatively severer clinical manifestation in females. They examined 4,736 consecutive CHF patients in stage $C / D$ (mean age 69 years), revealing that, compared with male patients, female patients were 3.8 years older and had lower prevalence of ischemic heart disease, diabetes, smoking, myocardial infarction (MI) and cancer. At baseline, females had more preserved left ventricular function but had higher NYHA functional class and increased brain natriuretic peptide levels. In females, aspirin, $\beta$-blockers and statins were less frequently used and diuretics were more frequently used. Crude mortality rate was comparable between the sexes during the median 3.1-year follow-up (52.4/1,000 and 47.3/1,000 person-year for females and males, respectively, $\mathrm{P}=0.225$ ). Multivariate Cox regression analysis revealed that females had a reduced risk of mortality (adjusted hazard ratio $0.791,95 \%$ confidence interval 0.640-0.979, $\mathrm{P}=0.031$ ). These results appear almost comparable with those in previous reports from Western countries, except the prevalence of hypertension was similar between the sexes in the present cohort, whereas in the previous reports, it was frequently observed in female HF patients.

To clarify the potential pathophysiological mechanisms underlying sex differences in heart failure, Meyer et al assessed sex-specific variation in clinical characteristics and biomarker levels in 567 patients (mean age $71 \pm 11$ years, 38\% female). Levels of biomarkers related to inflammation (C-reactive protein, pentraxin 3, growth differentiation factor 15 (GDF-15), and interleukin-6) and extracellular matrix remodeling (syndecan-1 and periostin) were significantly lower in women compared with men. They conclude that female HF patients have a distinct clinical presentation and better outcomes compared with male patients. In addition, the lower mortality was independent of differences in clinical characteristics, but differential sex associations between several biomarkers and mortality might partly explain the survival difference. ${ }^{9}$

Hara et $\mathrm{al}^{10}$ investigated the association between serum n-3 polyunsaturated fatty acids (n-3 PUFA) levels and HF events in 712 survivors of acute MI (AMI) using The Osaka Acute Coronary Insufficiency Study (OACIS), which is a prospective and observational study of AMI from the Osaka region of Japan
(ID: UMIN000004575). They clarified that low levels of circulating n-3 PUFA are associated with decreased HF-free survival in post-AMI patients, and the effect of low serum eicosapentaenoic acid level on HF hospitalization was prominent in male patients, suggesting a sex difference in HF after AMI.

There are a number of alternative potential explanations for the better outcomes in women with HF. The female heart appears to respond to injury differently from the male heart. For example, women have been reported to have less ventricular remodeling, preservation of right ventricular function, and protection against ventricular arrhythmias, neurohormonal activation, genetic mutations, myocyte necrosis, and apoptosis. ${ }^{11}$ Under stress, male hearts develop pathological hypertrophy with dilatation and poor systolic function more easily than female hearts. Women with aortic stenosis have concentric hypertrophy with better systolic function, less upregulation of extracellular matrix genes and better reversibility after unloading. Stressed female hearts maintain energy metabolism better than male hearts and are better protected against calcium overload. Estrogens and androgens and their receptors are present in the myocardium and lead to coordinated regulation of functionally relevant pathways. ${ }^{1}$

In conclusion, female CHF patients seems to have a better survival than males after adjustment for baseline differences, although the crude mortality rate is comparable between the sexes, possibly reflecting relatively severer clinical manifestations in females. By understanding that women are less likely to receive guideline-related $\mathrm{HF}$ treatment, but nevertheless do better than their male counterparts, guideline-related HF treatments may be promoted for female HF patients. Further investigation of sex differences in HF is warranted, leading to evidence-based medicine for HF in light of sex differences.

\section{References}

1. Regitz-Zagrosek V, Oertelt-Prigione S, Seeland U, Hetzer R. Sex and gender differences in myocardial hypertrophy and heart failure. Circ J 2010; 74: 1265-1273.

2. Yancy CW, Jessup M, Bozkurt B, Butler J, Casey DE Jr, Drazner $\mathrm{MH}$, et al. $2013 \mathrm{ACCF} / \mathrm{AHA}$ guideline for the management of heart failure: Executive summary: A report of the American College of Cardiology Foundation/American Heart Association Task Force on practice guidelines. Circulation 2013; 128: 1810-1852.

3. JCS Joint Working Group. Guidelines for Treatment of Acute Heart Failure (JCS 2011): Digest version. Circ J 2013; 77: 2157-2201

4. Ministry of Health, Labour and Welfare. http: //www.mhlw.go.jp/ english/.

5. O'Meara E, Clayton T, McEntegart MB, McMurray JJ, Pina IL, Granger CB, et al. Sex differences in clinical characteristics and prognosis in a broad spectrum of patients with heart failure: Results of the Candesartan in Heart failure: Assessment of Reduction in Mortality and morbidity (CHARM) program. Circulation 2007; 115: 3111-3120.

6. Martínez-Sellés M, Doughty RN, Poppe K, Whalley GA, Earle N, Tribouilloy C, et al. Meta-Analysis Global Group In Chronic Heart Failure (MAGGIC). Gender and survival in patients with heart failure: Interactions with diabetes and aetiology. Results from the MAGGIC individual patient meta-analysis. Eur J Heart Fail 2012; 14: 473-479.

7. Deswal A, Bozkurt B. Comparison of morbidity in women versus men with heart failure and preserved ejection fraction. Am J Cardiol 2006; 97: $1228-1231$

8. Sakata Y, Miyata S, Nochioka K, Miura M, Takada T, Tadaki S, et al. Gender differences in clinical characteristics, treatment and longterm outcome in patients with stage C/D heart failure in Japan: Report from the CHART-2 study. Circ J 2014; 78: 428-435.

9. Meyer S, van der Meer P, van Deursen VM, Jaarsma T, van Veldhuisen DJ, van der Wal MH, et al. Neurohormonal and clinical sex differences in heart failure. Eur Heart J 2013; 34: 2538-2547.

10. Hara M, Sakata Y, Nakatani D, Suna S, Usami M, Matsumoto S, et al. Low levels of serum n-3 polyunsaturated fatty acids are associated with worse heart failure-free survival in patients after acute myocardial infarction. Circ J 2013; 77: 153-162.

11. Martínez-Sellés M. What do women have in their hearts? Rev Esp Cardiol 2007; 60: 1118-1121. 